\title{
DUS Characterization of Narendra Lahar: A New High Yielding Rice Variety
}

\author{
S.P. Giri*, Alok Pandey, V.N. Singh, D.K. Verma, R.M. Tripathi, \\ Arun Kumar Singh, M.L. Maurya and R.V. Singh
}

\author{
Crop Research Station, Masodha (N.D. University of Agriculture and Technology), Post - \\ Dabhasemer, Faizabad -224133 (U.P.), India \\ *Corresponding author
}

\begin{tabular}{|c|c|}
\hline & A B S T R A C T \\
\hline Keywords & $\begin{array}{l}\text { Information regarding Novelty, distinctness, uniformity and stability are the basic } \\
\text { mandates for the protection of varieties under Protection of Plant Varieties and Farmers' }\end{array}$ \\
\hline $\begin{array}{l}\text { Yielding, } \\
\text { Uniformity, } \\
\text { Novelty, Varieties. }\end{array}$ & $\begin{array}{l}\text { Rights Act (PPVFRA), 2001. A field experiment was conducted at Crop Research Station, } \\
\text { Masodha Faizabad for DUS characterization of recently released high yielding rice variety } \\
\text { Narendra Lahar as per the guidelines of International Union for the Protection of New }\end{array}$ \\
\hline Article Info & $\begin{array}{l}\text { Varieties of Plants (UPOV) during kharit } 2015 \text {. DUS test results shows that Narendra } \\
\text { Lahar is medium maturing, maturing, good tillering ability and semi tall rice variety }\end{array}$ \\
\hline $\begin{array}{l}\text { Accepted: } \\
\text { 31 October } 2017 \\
\text { Available Online: } \\
10 \text { November } 2017\end{array}$ & $\begin{array}{l}\text { posses. The basal leaf sheath colour is green in coloration while leaf anthocynincolration is } \\
\text { absent in the variety. Leaf: pubescence of blade surface is very week and colorless auricles } \\
\text { were present in the variety. Ligule was present and green in color. The grain is long } \\
\text { slender in shape having intermediate amylase content. Molecular characterization using } \\
\text { SSR markers also exhibits distinctness of new variety. }\end{array}$ \\
\hline
\end{tabular}

\section{Introduction}

Rice is the staple food of more than $2 / 3^{\text {rd }}$ population of the world and one of the largest cultivated cereal crops. It is also the major source of nutrition and livelihood of more than 120-150 million rural households of the country and backbone of agriculture based economy of India. At 104 million tons, it accounts for $34 \%$ of food grain production and $41 \%$ of cereal production at present. At current rate of population growth of $1.8 \%$ per annum, the rice requirement of the country is estimated to be around 140-160 million tonnes by $2020(1,2,3)$. Achieving this target in the next few decades, without harming the environment would be a great challenge. Uttar Pradesh is the $2^{\text {nd }}$ largest producer of rice in the country just after West Bengal. Eastern part of the Uttar Pradesh is the hub of rice production and rice is grown in almost all the ecologies viz. rainfed upland, well managed irrigated and rainfed lowland due highly fertile soil of Indo - Gangetic plain. The major factors affecting rice productivity in this region is low coverage by ecosystem specific varieties/hybrids, degrading soil fertility/health, erratic mansoon rainfall, shortage of labours and timely supply of other inputs. Development of new rice variety specific to prevalent ecology and agro climatic conditions is one of the options to enhanced the productivity of this region $(4,5)$. In view of the above observation Narendra 
Lahar (NDR 370135) a new high yielding rice variety was developed at Crop Research Station, Masodha for favourable irrigated ecology of Uttar Pradesh. NDR 370135 was developed from triple cross of IR 68068-99-13-3-3/Janak//IRRI 105. This entry was tested in Regional Agriculture Demonstration \& Testing Station, Uttar Pradesh (RATDS) for consecutively three years from 20011-2014. The performance of NDR 370135 is promising in all the zones viz. central, eastern, Northern and western zones of Uttar Pradesh $(6,7,8)$. This variety was found resistant to major location specific pests viz. Stem borer and Leaf folder and also found moderately resistant to major diseases (Blast, Sheath Blight and Bacterial Leaf Blight). The head rice recovery (HRR) of Narendra Lahar was quantified $67.80 \%$. Agronomical evaluation of NDR 370135 indicated good fertilizer responsive trait of this rice variety. The yield capacity of this variety of irrigated medium duration is 55-60 q/ha. Performance of the NDR 370135 in On -Farm and Front Line Demonstartions was also very encouraging and it out yielded popular rice varieties of area in yield. NDR 370135 also established its yield superiority over most popular variety NDR 359 and may be good replacement for this variety as it has synchronous flowering which NDR 359 lacks. Information regarding Novelty, distinctness, uniformity and stability are basic requirement for the protection of varieties under Protection of Plant Varieties and Farmer's Rights ACT (PPVFRA), 2001. Hence, the molecular and morphological characteristics of the Narendra Lahar (NDR 370135) were studied for DUS characterization of the variety.

\section{Materials and Methods}

\section{DUS characterization of Narendra Lahar}

The DUS characterization of the Narendra Lahar (NDR 370135) was carried out at Crop Researcha Station, Masodha, Faizabad during kharif 2015. The soil of the experimental field was sandy loam in texture with very low organic carbon $(0.42 \%)$. The $\mathrm{pH}$ of the soil was found 7.2. Nitrogen content of the soil was $200 \mathrm{~kg} / \mathrm{ha}$, available $\mathrm{P} 2 \mathrm{O} 5$ is $24 \mathrm{~kg} / \mathrm{ha}$ and available $\mathrm{K} 2 \mathrm{O}$ was $234 \mathrm{~kg} / \mathrm{ha}$. The experiment was laid down in randomized block design with three replications. The plot size of the experimental plots was $15 \mathrm{sqm}$ and spacing of $20 \mathrm{~cm}$ between rows and $15 \mathrm{~cm}$ between plants was maintained to grow the crop. Recommended package of practices were adopted to raise the crop. Observations on agro morphological traits were recorded at different growth stages of the crop for the DUS characterization of the Variety as per the national guidelines for DUS test in rice (Table $1)$.

\section{Molecular characterization of Narendra Lahar}

\section{DNA isolation and PCR assays}

The genomic DNA was extracted from 10 days old rice seedlings as per Dellaporta $e t$ al., (1993). PCR analysis was performed with 0.2 ilTaq DNA polymerase (5U/il) (Biotools), 1 il of genomic DNA $10 \mathrm{ng} / \mathrm{il}, 1$ il of $10 \mathrm{X}$ buffer (Biotools), 0.5 il of dNTPs $(2.5 \mathrm{mM})$ and 1il of each primer pair in a total volume of $10 \mathrm{il}$.

PCR was performed using thermal cycler (Applied Biosystems) following the PCR protocols reported earlier with necessary modifications. The amplified products were separated in 3\% metaphor gel and visualized under phospho imager system after staining with ethidium bromide. The sizes of the amplified fragments were estimated visually using 100bp DNA ladder as size standard. Only clear and unambiguous bands of markers were scored. Band position of the prominent markers was depicted in table 2 and figure 1. 
Table.1 DUS characteristics of Narendra Lahar (NDR 370135)

\begin{tabular}{|c|c|c|c|c|c|}
\hline S.No. & Characteristics & States & Note & $\begin{array}{c}\text { Stage of } \\
\text { observation }\end{array}$ & $\begin{array}{l}\text { Type of } \\
\text { assessment }\end{array}$ \\
\hline 1 & Coleoptile: colour & $\begin{array}{l}\text { Colourless } \\
\text { Green } \\
\text { Purple }\end{array}$ & $\begin{array}{l}1 \\
2 \\
3\end{array}$ & Germination & 1 \\
\hline $\begin{array}{l}2 . \\
(*)\end{array}$ & Basal leaf: sheath colour & $\begin{array}{l}\text { Green } \\
\text { Light purple } \\
\text { Purple lines } \\
\text { Purple }\end{array}$ & $\begin{array}{l}1 \\
2 \\
3 \\
4\end{array}$ & $\begin{array}{l}\text { Vegetative } \\
\text { growth }\end{array}$ & 1 \\
\hline 3 & $\begin{array}{l}\text { Leaf: intensity of green } \\
\text { colour }\end{array}$ & $\begin{array}{l}\text { Light } \\
\text { Medium } \\
\text { Dark }\end{array}$ & $\begin{array}{l}3 \\
5 \\
7\end{array}$ & $\begin{array}{l}\text { Vegetative } \\
\text { growth }\end{array}$ & 5 \\
\hline 4 & $\begin{array}{l}\text { Leaf: anthocyanin } \\
\text { colouration }\end{array}$ & $\begin{array}{l}\text { Absent } \\
\text { Present }\end{array}$ & $\begin{array}{l}1 \\
9\end{array}$ & $\begin{array}{l}\text { Vegetative } \\
\text { growth }\end{array}$ & 1 \\
\hline 5 & $\begin{array}{l}\text { Leaf : distribution of } \\
\text { anthocyanin colouration }\end{array}$ & $\begin{array}{l}\text { On tips only } \\
\text { On margins only } \\
\text { In blotches only } \\
\text { Uniform }\end{array}$ & $\begin{array}{l}1 \\
2 \\
3 \\
4 \\
\end{array}$ & $\begin{array}{l}\text { Vegetative } \\
\text { growth }\end{array}$ & 3 \\
\hline 6 & $\begin{array}{l}\text { Leaf sheath: anthocyanin } \\
\text { colouration }\end{array}$ & $\begin{array}{l}\text { Absent } \\
\text { Present }\end{array}$ & $\begin{array}{l}1 \\
9\end{array}$ & $\begin{array}{l}\text { Vegetative } \\
\text { growth }\end{array}$ & 1 \\
\hline 7 & $\begin{array}{l}\text { Leaf sheath : intensity of } \\
\text { anthocyanin colouration }\end{array}$ & $\begin{array}{l}\text { Very weak } \\
\text { Weak } \\
\text { Medium } \\
\text { Strong } \\
\text { Very strong }\end{array}$ & $\begin{array}{l}1 \\
3 \\
5 \\
7 \\
9\end{array}$ & $\begin{array}{l}\text { Vegetative } \\
\text { growth }\end{array}$ & 1 \\
\hline $\begin{array}{c}8 \\
(*)\end{array}$ & $\begin{array}{l}\text { Leaf: pubescence of blade } \\
\text { surface }\end{array}$ & $\begin{array}{l}\text { Absent } \\
\text { Weak } \\
\text { Medium } \\
\text { Strong } \\
\text { Very strong }\end{array}$ & $\begin{array}{l}1 \\
3 \\
5 \\
7 \\
9\end{array}$ & $\begin{array}{l}\text { Vegetative } \\
\text { growth }\end{array}$ & 3 \\
\hline $\begin{array}{c}9 \\
(*)\end{array}$ & Leaf : auricles & $\begin{array}{l}\text { Absent } \\
\text { Present }\end{array}$ & $\begin{array}{l}1 \\
9\end{array}$ & $\begin{array}{l}\text { Vegetative } \\
\text { growth }\end{array}$ & 9 \\
\hline $\begin{array}{l}10 \\
(*)\end{array}$ & $\begin{array}{l}\text { Leaf: anthocyanin } \\
\text { colouration of auricles }\end{array}$ & $\begin{array}{l}\text { Colourless } \\
\text { Light purple } \\
\text { Purple }\end{array}$ & $\begin{array}{l}1 \\
2 \\
3 \\
\end{array}$ & $\begin{array}{l}\text { Vegetative } \\
\text { growth }\end{array}$ & 1 \\
\hline 11 & Leaf: collar & $\begin{array}{l}\text { Absent } \\
\text { Present }\end{array}$ & $\begin{array}{l}1 \\
9 \\
\end{array}$ & $\begin{array}{l}\text { Vegetative } \\
\text { growth }\end{array}$ & 9 \\
\hline 12 & $\begin{array}{l}\text { Leaf: anthocyanin } \\
\text { colouration of collar }\end{array}$ & $\begin{array}{l}\text { Absent } \\
\text { Present }\end{array}$ & $\begin{array}{l}1 \\
9\end{array}$ & $\begin{array}{l}\text { Vegetative } \\
\text { growth }\end{array}$ & 1 \\
\hline 13 & Leaf: ligule & $\begin{array}{l}\text { Absent } \\
\text { Present }\end{array}$ & $\begin{array}{l}1 \\
9 \\
\end{array}$ & $\begin{array}{l}\text { Vegetative } \\
\text { growth }\end{array}$ & 9 \\
\hline $\begin{array}{l}14 \\
(*)\end{array}$ & Leaf: shape of ligule & $\begin{array}{l}\text { Truncate } \\
\text { Acute } \\
\text { Split }\end{array}$ & $\begin{array}{l}1 \\
2 \\
3\end{array}$ & $\begin{array}{l}\text { Vegetative } \\
\text { growth }\end{array}$ & 1 \\
\hline $\begin{array}{r}15 \\
(*) .\end{array}$ & Leaf: colour of ligule & $\begin{array}{l}\text { Green } \\
\text { Light purple } \\
\text { Purple }\end{array}$ & $\begin{array}{l}1 \\
2 \\
3\end{array}$ & $\begin{array}{l}\text { Vegetative } \\
\text { growth }\end{array}$ & 1 \\
\hline 16 & Leaf: length of blade & $\begin{array}{l}\text { Short } \\
\text { Medium } \\
\text { Long }\end{array}$ & $\begin{array}{l}3 \\
5 \\
7\end{array}$ & $\begin{array}{l}\text { Vegetative } \\
\text { growth }\end{array}$ & 3 \\
\hline 17 & Leaf: width of blade & $\begin{array}{l}\text { Narrow } \\
\text { Medium } \\
\text { Broad }\end{array}$ & $\begin{array}{l}3 \\
5 \\
7\end{array}$ & $\begin{array}{l}\text { Vegetative } \\
\text { growth }\end{array}$ & 5 \\
\hline
\end{tabular}




\begin{tabular}{|c|c|c|c|c|c|}
\hline S.No. & Characteristics & States & Note & $\begin{array}{c}\text { Stage of } \\
\text { observation }\end{array}$ & $\begin{array}{l}\text { Type of } \\
\text { assessment }\end{array}$ \\
\hline 18 & $\begin{array}{l}\text { Culm: attitude (for floating } \\
\text { rice only) }\end{array}$ & $\begin{array}{l}\text { Non procumbent } \\
\text { Procumbent }\end{array}$ & $\begin{array}{l}1 \\
9\end{array}$ & $\begin{array}{l}\text { Vegetative } \\
\text { growth }\end{array}$ & 1 \\
\hline $\begin{array}{l}19 \\
(+)\end{array}$ & Culm: attitude & $\begin{array}{l}\text { Erect } \\
\text { Semi-erect } \\
\text { Open } \\
\text { Spreading } \\
\end{array}$ & $\begin{array}{l}1 \\
3 \\
5 \\
7\end{array}$ & $\begin{array}{l}\text { Vegetative } \\
\text { growth }\end{array}$ & 1 \\
\hline $\begin{array}{l}20 \\
(*)\end{array}$ & $\begin{array}{l}\text { Time of heading ( } 50 \% \text { of } \\
\text { plants with panicles) }\end{array}$ & $\begin{array}{l}\text { Very early ( }<71 \text { days) } \\
\text { Early ( } 71-90 \text { days) } \\
\text { Medium ( } 91-110 \text { days) } \\
\text { Late (111-130 days) } \\
\text { Very late (>130 days) }\end{array}$ & $\begin{array}{l}1 \\
3 \\
5 \\
7 \\
9 \\
\end{array}$ & $\begin{array}{l}\text { Vegetative } \\
\text { growth }\end{array}$ & 5 \\
\hline $\begin{array}{l}21 \\
(*) \\
(+)\end{array}$ & $\begin{array}{l}\text { Flag leaf: attitude of blade } \\
\text { (early observation) }\end{array}$ & $\begin{array}{l}\text { erect } \\
\text { semi-erect } \\
\text { horizontal } \\
\text { deflexed }\end{array}$ & $\begin{array}{l}1 \\
3 \\
5 \\
7\end{array}$ & $\begin{array}{l}\text { Vegetative } \\
\text { growth }\end{array}$ & 1 \\
\hline $\begin{array}{l}22 \\
(*)\end{array}$ & $\begin{array}{l}\text { Spikelet: density of } \\
\text { pubescence of lemma }\end{array}$ & $\begin{array}{l}\text { Absent } \\
\text { Weak } \\
\text { Medium } \\
\text { Strong } \\
\text { Very strong } \\
\end{array}$ & $\begin{array}{l}1 \\
3 \\
5 \\
7 \\
9 \\
\end{array}$ & $\begin{array}{l}\text { Reproductive } \\
\text { stage }\end{array}$ & 1 \\
\hline 23 & Male sterility & $\begin{array}{l}\text { Absent } \\
\text { Present }\end{array}$ & $\begin{array}{l}1 \\
9\end{array}$ & $\begin{array}{l}\text { Reproductive } \\
\text { stage }\end{array}$ & 1 \\
\hline 24 & $\begin{array}{l}\text { Lemma: anthocyanin } \\
\text { colouration of keel }\end{array}$ & $\begin{array}{l}\text { Absent or very weak } \\
\text { Weak } \\
\text { Medium } \\
\text { Strong } \\
\text { Very strong }\end{array}$ & $\begin{array}{l}1 \\
3 \\
5 \\
7 \\
9 \\
\end{array}$ & $\begin{array}{l}\text { Reproductive } \\
\text { stage }\end{array}$ & 1 \\
\hline 25 & $\begin{array}{l}\text { Lemma: anthocyanin } \\
\text { colouration of area below } \\
\text { apex }\end{array}$ & $\begin{array}{l}\text { Absent } \\
\text { Weak } \\
\text { Medium } \\
\text { Strong } \\
\text { Very strong }\end{array}$ & $\begin{array}{l}1 \\
3 \\
5 \\
7 \\
9\end{array}$ & $\begin{array}{l}\text { Reproductive } \\
\text { stage }\end{array}$ & 1 \\
\hline $\begin{array}{l}26 \\
(*)\end{array}$ & $\begin{array}{l}\text { Lemma: anthocyanin } \\
\text { colouration of apex }\end{array}$ & $\begin{array}{l}\text { Absent } \\
\text { Weak } \\
\text { Medium } \\
\text { Strong } \\
\text { Very strong } \\
\end{array}$ & $\begin{array}{l}1 \\
3 \\
5 \\
7 \\
9 \\
\end{array}$ & $\begin{array}{l}\text { Reproductive } \\
\text { stage }\end{array}$ & 1 \\
\hline $\begin{array}{l}27 \\
(*)\end{array}$ & Spikelet: colour of stigma & $\begin{array}{l}\text { White } \\
\text { Light green } \\
\text { Yellow } \\
\text { Light purple } \\
\text { Purple } \\
\end{array}$ & $\begin{array}{l}1 \\
2 \\
3 \\
4 \\
5\end{array}$ & $\begin{array}{l}\text { Reproductive } \\
\text { stage }\end{array}$ & 1 \\
\hline $\begin{array}{l}28 \\
(+)\end{array}$ & Stem: thickness & $\begin{array}{l}\text { Thin } \\
\text { Medium } \\
\text { Thick }\end{array}$ & $\begin{array}{l}3 \\
5 \\
7\end{array}$ & Maturity & 7 \\
\hline $\begin{array}{l}29 \\
(*)\end{array}$ & $\begin{array}{l}\text { Stem: length (excluding } \\
\text { panicle; excluding floating } \\
\text { rice) }\end{array}$ & $\begin{array}{l}\text { Very short }(<91 \mathrm{~cm}) \\
\text { Short }(91-110 \mathrm{~cm}) \\
\text { Medium }(111-130 \mathrm{~cm}) \\
\text { Long }(131-150 \mathrm{~cm}) \\
\text { Very long }(>150 \mathrm{~cm}) \\
\end{array}$ & $\begin{array}{l}1 \\
3 \\
5 \\
7 \\
9 \\
\end{array}$ & Maturity & 5 \\
\hline $\begin{array}{l}30 \\
(*) \\
\end{array}$ & $\begin{array}{l}\text { Stem: anthocyanin } \\
\text { colouration of nodes }\end{array}$ & $\begin{array}{l}\text { Absent } \\
\text { Present }\end{array}$ & $\begin{array}{l}1 \\
9\end{array}$ & Maturity & 1 \\
\hline
\end{tabular}




\begin{tabular}{|c|c|c|c|c|c|}
\hline S.No. & Characteristics & States & Note & $\begin{array}{c}\text { Stage of } \\
\text { observation }\end{array}$ & $\begin{array}{l}\text { Type of } \\
\text { assessment }\end{array}$ \\
\hline 31 & $\begin{array}{l}\text { Stem : intensity of } \\
\text { anthocyanin colouration of } \\
\text { nodes }\end{array}$ & $\begin{array}{l}\text { Weak } \\
\text { Medium } \\
\text { Strong }\end{array}$ & $\begin{array}{l}3 \\
5 \\
7\end{array}$ & Maturity & \\
\hline 32 & $\begin{array}{l}\text { Stem: anthocyanin } \\
\text { colouration of internodes }\end{array}$ & $\begin{array}{l}\text { Absent } \\
\text { Present }\end{array}$ & $\begin{array}{l}1 \\
9\end{array}$ & Maturity & 1 \\
\hline $\begin{array}{l}33 \\
(*) \\
(+)\end{array}$ & $\begin{array}{l}\text { Panicle: length of main } \\
\text { axis }\end{array}$ & $\begin{array}{l}\text { Very short }(<16 \mathrm{~cm}) \\
\text { Short }(16-20 \mathrm{~cm}) \\
\text { Medium }(21-25 \mathrm{~cm}) \\
\text { Long }(26-30 \mathrm{~cm}) \\
\text { Very long }(>30 \mathrm{~cm}) \\
\end{array}$ & $\begin{array}{l}1 \\
3 \\
5 \\
7 \\
9 \\
\end{array}$ & Maturity & 7 \\
\hline $\begin{array}{l}34 \\
(*) \\
(+)\end{array}$ & $\begin{array}{l}\text { Flag leaf: attitude of blade } \\
\text { (late observation) }\end{array}$ & $\begin{array}{l}\text { Erect } \\
\text { Semi-erect } \\
\text { Horizontal } \\
\text { Deflexed }\end{array}$ & $\begin{array}{l}1 \\
3 \\
5 \\
7\end{array}$ & Maturity & 1 \\
\hline $\begin{array}{l}35 \\
(*) \\
(+)\end{array}$ & $\begin{array}{l}\text { Panicle: curvature of main } \\
\text { axis }\end{array}$ & $\begin{array}{l}\text { Straight } \\
\text { Semi-straight } \\
\text { Drooping } \\
\text { Deflexed } \\
\end{array}$ & $\begin{array}{l}1 \\
3 \\
5 \\
7\end{array}$ & Maturity & 1 \\
\hline 36 & Panicle: number per plant & $\begin{array}{l}\text { Few }(<11) \\
\text { Medium }(11-20) \\
\text { Many }(>20)\end{array}$ & $\begin{array}{l}3 \\
5 \\
7\end{array}$ & Maturity & 5 \\
\hline $\begin{array}{l}37 \\
(*)\end{array}$ & $\begin{array}{l}\text { Spikelet : colour of tip of } \\
\text { lemma }\end{array}$ & $\begin{array}{l}\text { White } \\
\text { Yellowish } \\
\text { Brown } \\
\text { Red } \\
\text { Purple } \\
\text { Black } \\
\end{array}$ & $\begin{array}{l}1 \\
2 \\
3 \\
4 \\
5 \\
6\end{array}$ & Maturity & 1 \\
\hline 38 & Lemma and Palea: colour & $\begin{array}{l}\text { Straw } \\
\text { Gold and gold furrows on } \\
\text { straw background } \\
\text { Brown spots on straw } \\
\text { Brown furrows on straw } \\
\text { Brown (tawny) } \\
\text { Reddish to light purple } \\
\text { Purple spots on straw } \\
\text { Purple furrows on straw } \\
\text { Purple } \\
\text { Black }\end{array}$ & $\begin{array}{c}1 \\
2 \\
\\
3 \\
4 \\
5 \\
6 \\
7 \\
8 \\
9 \\
10\end{array}$ & Maturity & 1 \\
\hline $\begin{array}{l}39 \\
(*) \\
\end{array}$ & Panicle : awns & $\begin{array}{l}\text { Absent } \\
\text { Present }\end{array}$ & $\begin{array}{l}1 \\
9 \\
\end{array}$ & Maturity & 1 \\
\hline $\begin{array}{l}40 \\
(*)\end{array}$ & $\begin{array}{l}\text { Panicle: colour of awns } \\
\text { (late observation) }\end{array}$ & $\begin{array}{l}\text { Yellowish white } \\
\text { Yellowish brown } \\
\text { Brown } \\
\text { Reddish brown } \\
\text { Light red } \\
\text { Red } \\
\text { Light purple } \\
\text { Purple } \\
\text { Black }\end{array}$ & $\begin{array}{l}1 \\
2 \\
3 \\
4 \\
5 \\
6 \\
7 \\
8 \\
9\end{array}$ & Maturity & - \\
\hline 41 & $\begin{array}{l}\text { Panicle: length of longest } \\
\text { awn }\end{array}$ & $\begin{array}{l}\text { Very short } \\
\text { Short } \\
\text { Medium } \\
\text { Long }\end{array}$ & $\begin{array}{l}1 \\
3 \\
5 \\
7\end{array}$ & Maturity & - \\
\hline
\end{tabular}




\begin{tabular}{|c|c|c|c|c|c|}
\hline S.No. & Characteristics & States & Note & $\begin{array}{c}\text { Stage of } \\
\text { observation }\end{array}$ & $\begin{array}{l}\text { Type of } \\
\text { assessment }\end{array}$ \\
\hline & & Very long & 9 & & \\
\hline $\begin{array}{l}42 \\
(*)\end{array}$ & $\begin{array}{l}\text { Panicle: distribution of } \\
\text { awns }\end{array}$ & $\begin{array}{l}\text { Tip only } \\
\text { Upper half only Whole } \\
\text { length }\end{array}$ & $\begin{array}{l}1 \\
3 \\
5\end{array}$ & Maturity & - \\
\hline $\begin{array}{l}43 \\
(+)\end{array}$ & $\begin{array}{l}\text { Panicle: presence of } \\
\text { secondary branching }\end{array}$ & $\begin{array}{l}\text { Absent } \\
\text { Present }\end{array}$ & $\begin{array}{l}1 \\
9\end{array}$ & Maturity & 9 \\
\hline $\begin{array}{l}44 . \\
(+)\end{array}$ & $\begin{array}{l}\text { Panicle: secondary } \\
\text { branching }\end{array}$ & $\begin{array}{l}\text { Weak } \\
\text { Strong } \\
\text { Clustered }\end{array}$ & $\begin{array}{l}1 \\
2 \\
3\end{array}$ & Maturity & 1 \\
\hline $\begin{array}{l}45 \\
(*) \\
(+)\end{array}$ & $\begin{array}{l}\text { Panicle: attitude of } \\
\text { branches }\end{array}$ & $\begin{array}{l}\text { Erect } \\
\text { Erect to semi-erect } \\
\text { Semi-erect } \\
\text { Semi-erect to spreading } \\
\text { Spreading }\end{array}$ & $\begin{array}{l}1 \\
3 \\
5 \\
7 \\
9\end{array}$ & Maturity & 3 \\
\hline $\begin{array}{c}46(*) \\
(+)\end{array}$ & Panicle: exsertion & $\begin{array}{l}\text { Partly exserted } \\
\text { Exserted } \\
\text { Well exserted }\end{array}$ & $\begin{array}{l}3 \\
5 \\
7\end{array}$ & Maturity & 7 \\
\hline 47 & Time of maturity & $\begin{array}{l}\text { Very early } \\
\text { Early } \\
\text { Medium } \\
\text { Late } \\
\text { Very late }\end{array}$ & $\begin{array}{l}1 \\
3 \\
5 \\
7 \\
9\end{array}$ & Maturity & 5 \\
\hline $\begin{array}{l}48 \\
(+)\end{array}$ & Leaf: senescence & $\begin{array}{l}\text { Early } \\
\text { Medium } \\
\text { Late }\end{array}$ & $\begin{array}{l}3 \\
5 \\
7\end{array}$ & Maturity & 5 \\
\hline $\begin{array}{l}49 \\
(*)\end{array}$ & Sterile lemma: colour & $\begin{array}{l}\text { Straw } \\
\text { Gold } \\
\text { Red } \\
\text { Purple }\end{array}$ & $\begin{array}{l}1 \\
2 \\
3 \\
4\end{array}$ & Maturity & 1 \\
\hline 50 & $\begin{array}{l}\text { Grain: weight of } 1000 \\
\text { fully developed grains }\end{array}$ & $\begin{array}{l}\text { Very low } \\
\text { Low } \\
\text { Medium } \\
\text { High } \\
\text { Very high }\end{array}$ & $\begin{array}{l}1 \\
3 \\
5 \\
7 \\
9\end{array}$ & Maturity & 5 \\
\hline $\begin{array}{l}51 \\
(+)\end{array}$ & Grain: length & $\begin{array}{l}\text { Very short } \\
\text { Short } \\
\text { Medium } \\
\text { Long } \\
\text { Very long } \\
\end{array}$ & $\begin{array}{l}1 \\
3 \\
5 \\
7 \\
9\end{array}$ & Maturity & 7 \\
\hline $\begin{array}{l}52 \\
(+)\end{array}$ & Grain: width & $\begin{array}{l}\text { Very narrow } \\
\text { Narrow } \\
\text { Medium } \\
\text { Broad } \\
\text { Very broad } \\
\end{array}$ & $\begin{array}{l}1 \\
3 \\
5 \\
7 \\
9 \\
\end{array}$ & Maturity & 5 \\
\hline $\begin{array}{l}53 \\
(+) \\
\end{array}$ & $\begin{array}{l}\text { Grain: phenol reaction of } \\
\text { lemma }\end{array}$ & $\begin{array}{l}\text { Absent } \\
\text { Present }\end{array}$ & $\begin{array}{l}1 \\
9 \\
\end{array}$ & Maturity & 1 \\
\hline $\begin{array}{l}54 \\
(*)\end{array}$ & Decorticated grain: length & $\begin{array}{l}\text { Very short } \\
\text { Short } \\
\text { Medium } \\
\text { Long } \\
\text { Very long } \\
\end{array}$ & $\begin{array}{l}1 \\
3 \\
5 \\
7 \\
9\end{array}$ & Maturity & 7 \\
\hline
\end{tabular}




\begin{tabular}{|c|c|c|c|c|c|}
\hline S.No. & Characteristics & States & Note & $\begin{array}{c}\text { Stage of } \\
\text { observation }\end{array}$ & $\begin{array}{l}\text { Type of } \\
\text { assessment }\end{array}$ \\
\hline $\begin{array}{l}55 \\
(*)\end{array}$ & Decorticated grain: width & $\begin{array}{l}\text { Narrow }(<2.0 \mathrm{~mm}) \\
\text { Medium }(2.0-2.5 \mathrm{~mm}) \\
\text { Broad }(>2.5 \mathrm{~mm})\end{array}$ & $\begin{array}{l}3 \\
5 \\
7\end{array}$ & Maturity & 5 \\
\hline $\begin{array}{l}56 . \\
(*) \\
(+)\end{array}$ & $\begin{array}{l}\text { Decorticated grain: shape } \\
\text { (in lateral view) }\end{array}$ & $\begin{array}{l}\text { Short slender } \\
\text { Short bold } \\
\text { Medium slender } \\
\text { Long slender } \\
\text { Long bold } \\
\text { Extra long slender }\end{array}$ & $\begin{array}{l}1 \\
2 \\
3 \\
4 \\
5 \\
6\end{array}$ & Maturity & 4 \\
\hline $\begin{array}{l}57 \\
(*)\end{array}$ & Decorticated grain: colour & $\begin{array}{l}\text { White } \\
\text { Light brown } \\
\text { Variegated brown } \\
\text { Dark brown } \\
\text { Light red } \\
\text { Red } \\
\text { Variegated purple } \\
\text { Purple } \\
\text { Dark purple }\end{array}$ & $\begin{array}{l}1 \\
2 \\
3 \\
4 \\
5 \\
6 \\
7 \\
7 \\
8 \\
9\end{array}$ & Maturity & 1 \\
\hline $\begin{array}{l}58 . \\
(+)\end{array}$ & $\begin{array}{l}\text { Endosperm: presence of } \\
\text { amylose }\end{array}$ & $\begin{array}{l}\text { Absent } \\
\text { Present }\end{array}$ & $\begin{array}{l}1 \\
9\end{array}$ & Maturity & 9 \\
\hline $\begin{array}{l}59 \\
(*) \\
(+)\end{array}$ & $\begin{array}{l}\text { Endosperm: content of } \\
\text { amylose }\end{array}$ & $\begin{array}{l}\text { Very low }(<10 \%) \\
\text { Low }(10-19 \%) \\
\text { Medium }(20-25 \%) \\
\text { High }(26-30 \%) \\
\text { Very high }(>30 \%)\end{array}$ & $\begin{array}{l}1 \\
3 \\
5 \\
7 \\
9\end{array}$ & Maturity & 5 \\
\hline $\begin{array}{l}60 . \\
(+)\end{array}$ & $\begin{array}{l}\text { Varieties with endosperm } \\
\text { of amylose absent only } \\
\text { Polished grain : expression } \\
\text { of white core }\end{array}$ & $\begin{array}{l}\text { Absent or very small } \\
\text { Smal } \\
\text { Medium } \\
\text { Large }\end{array}$ & $\begin{array}{l}1 \\
3 \\
5 \\
7\end{array}$ & Maturity & - \\
\hline $\begin{array}{l}61 \\
(+)\end{array}$ & $\begin{array}{l}\text { Gelatinization temperature } \\
\text { through alkali spreading } \\
\text { value. }\end{array}$ & $\begin{array}{l}\text { Low } \\
\text { Medium } \\
\text { High medium } \\
\text { High }\end{array}$ & $\begin{array}{l}1 \\
3 \\
5 \\
7\end{array}$ & Maturity & 3 \\
\hline $\begin{array}{l}62 \\
(*) \\
(+)\end{array}$ & Decorticated grain: aroma & $\begin{array}{l}\text { Absent } \\
\text { Present }\end{array}$ & $\begin{array}{l}1 \\
9\end{array}$ & Maturity & 1 \\
\hline
\end{tabular}

Table.2 Band position of prominent markers in Narendra Lahar

\begin{tabular}{|c|l|l|l|l|}
\hline SN. & Markers & \multicolumn{1}{|c|}{ Forward Sequence } & \multicolumn{1}{c|}{ Reverse Sequence } & bp \\
\hline $\mathbf{1}$ & RM495 & AATCCAAGGTGCAGAGATGG & CAACGATGACGAACACAACC & 140 \\
\hline $\mathbf{2}$ & RM1 & GCGAAAACACAATGCAAAAA & GCGTTGGTTGGACCTGAC & 110 \\
\hline $\mathbf{3}$ & RM283 & GGCATGAGAGTCTGTGATGTTGG & TAGTACTGCTCCATCTGCCTTGG & 160 \\
\hline $\mathbf{4}$ & RM259 & TGGAGTTTGAGAGGAGGG & CTTGTTGCATGGTGCCATGT & 100 \\
\hline $\mathbf{5}$ & RM312 & GTATGCATATTTGATAAGAG & AAGTCACCGAGTTTACCTTC & 100 \\
\hline $\mathbf{6}$ & RM5 & TGCAACTTCTAGCTGCTCGA & GCATCCGATCTTGATGGG & 110 \\
\hline $\mathbf{7}$ & RM431 & GCTTGCTTGTATCTGCATTGGTAGG & GGGATGATCCACTCTCTGTTTGG & 260 \\
\hline $\mathbf{8}$ & RM154 & GACGGTGACGCACTTTATGAACC & CGATCTGCGAGAAACCCTCTCC & 170 \\
\hline $\mathbf{9}$ & RM452 & CTGATCGAGAGCGTTAAGGG & GGGATCAAACCACGTTTCTG & 230 \\
\hline
\end{tabular}




\begin{tabular}{|c|c|c|c|c|}
\hline SN. & Markers & Forward Sequence & Reverse Sequence & bp \\
\hline 10 & RM489 & ACTTGAGACGATCGGACACC & TCACCCATGGATGTTGTCAG & 250 \\
\hline 11 & OSR-13 & CATTTGTGCGTCACGGAGTA & AGCCACAGCGCCCATCTCTC & 100 \\
\hline 12 & RM338 & CACAGGAGCAGGAGAAGAGC & GGCAAACCGATCACTCAGTC & 200 \\
\hline 13 & RM55 & CCGTCGCCGTAGTAGAGAAG & TCCCGGTTATTTTAAGGCG & 250 \\
\hline 14 & RM514 & AGATTGATCTCCCATTCCCC & CACGAGCATATTACTAGTGG & 280 \\
\hline 15 & RM307 & GTACTACCGACCTACCGTTCAC & CTGCTATGCATGAACTGCTC & 150 \\
\hline 16 & RM124 & ATCGTCTGCGTTGCGGCTGCTG & CATGGATCACCGAGCTCCCCCC & 300 \\
\hline 17 & RM507 & TGCCCATGTATGTGAGGTACTCC & GCCTAATCCAGGACAAGCTACGG & 300 \\
\hline 18 & RM413 & CCAATCTTGTCTTCCGGATCTTGC & AGATAGCCATGGGCGATTCTTGG & 90 \\
\hline 19 & RM161 & TGCAGATGAGAAGCGGCGCCTC & TGTGTCATCAGACGGCGCTCCG & 200 \\
\hline 20 & RM178 & TCGCGTGAAAGATAAGCGGCGC & GATCACCGTTCCCTCCGCCTGC & 140 \\
\hline 21 & RM334 & GTTCAGTGTTCAGTGCCACC & GACTTTGATCTTTGGTGGACG & 200 \\
\hline 22 & RM133 & TTGGATTGTTTTGCTGGCTCGC & GGAACACGGGGTCGGAAGCGAC & 250 \\
\hline 23 & RM510 & AACCGGATTAGTTTCTCGCC & TGAGGACGACGAGCAGATTC & 150 \\
\hline 24 & RM454 & CTCAAGCTTAGCTGCTGCTG & GTGATCAGTGCACCATAGCG & 300 \\
\hline 25 & RM162 & GCCAGCAAAACCAGGGATCCGG & CAAGGTCTTGTGCGGCTTGCGG & 250 \\
\hline 26 & RM125 & ATCAGCAGCCATGGCAGCGACC & AGGGGATCATGTGCCGAAGGCC & 110 \\
\hline 27 & RM11 & TCTCCTCTTCCCCCGATC & ATAGCGGGCGAGGCTTAG & 150 \\
\hline 28 & RM455 & CCACAAATTAATCCGGATCACACC & AGCATTGTGCAATCACGAGAAGG & 120 \\
\hline 29 & RM118 & CCAATCGGAGCCACCGGAGAGC & CACATCCTCCAGCGACGCCGAG & 150 \\
\hline 30 & RM408 & CAACGAGCTAACTTCCGTCC & ACTGCTACTTGGGTAGCTGACC & 120 \\
\hline 31 & RM152 & GAAACCACCACACCTCACCG & CCGTAGACCTTCTTGAAGTAG & 150 \\
\hline 32 & RM44 & ACGGGCAATCCGAACAACC & TCGGGAAAACCTACCCTACC & 110 \\
\hline 33 & RM284 & ACTGCATGATCCTCCTCAGATCC & СССТCTGATCTCTGATACTCCATCC & 140 \\
\hline 34 & RM433 & TGCGCTGAACTAAACACAGC & AGACAAACCTGGCCATTCAC & 250 \\
\hline 35 & RM447 & CCCTTGTGCTGTCTCCTCTC & ACGGGCTTCTTCTCCTTCTC & 110 \\
\hline 36 & RM316 & CTAGTTGGGCATACGATGGC & ACGCTTATATGTTACGTCAAC & 230 \\
\hline 37 & RM105 & GTCGTCGACCCATCGGAGCCAC & TGGTCGAGGTGGGGATCGGGTC & 130 \\
\hline 38 & RM215 & CAAAATGGAGCAGCAAGAGC & TGAGCACCTCCTTCTCTGTAG & 150 \\
\hline 39 & RM474 & AAGATGTACGGGTGGCATTC & TATGAGCTGGTGAGCAATGG & 250 \\
\hline 40 & RM271 & TCAGATCTACAATTCCATCC & TCGGTGAGACCTAGAGAGCC & 110 \\
\hline 41 & RM171 & AACGCGAGGACACGTACTTAC & ACGAGATACGTACGCCTTTG & 320 \\
\hline 42 & RM484 & TCTCССТССТСАССАTТGTC & TGCTGCCCTCTCTCTCTCTC & 300 \\
\hline 43 & RM552 & CGCAGTTGTGGATTTCAGTG & TGCTCAACGTTTGACTGTCC & 180 \\
\hline 44 & RM536 & TCTCTCCTCTTGTTTGGCTC & ACACACCAACACGACCACAC & 120 \\
\hline 45 & RM287 & TTCCCTGTTAAGAGAGAAATC & GTGTATTTGGTGAAAGCAAC & 120 \\
\hline 46 & RM144 & TGCCCTGGCGCAAATTTGATCC & GCTAGAGGAGATCAGATGGTAGTGCATG & 250 \\
\hline 47 & RM19 & CAAAAACAGAGCAGATGAC & CTCAAGATGGACGCCAAGA & 260 \\
\hline 48 & RM277 & CGGTCAAATCATCACCTGAC & CAAGGCTTGCAAGGGAAG & 140 \\
\hline
\end{tabular}


Fig.1 Major Markers and Band positions in Narendra Lahar (NDR 370135)

NDR-370135 RM495 to RM277

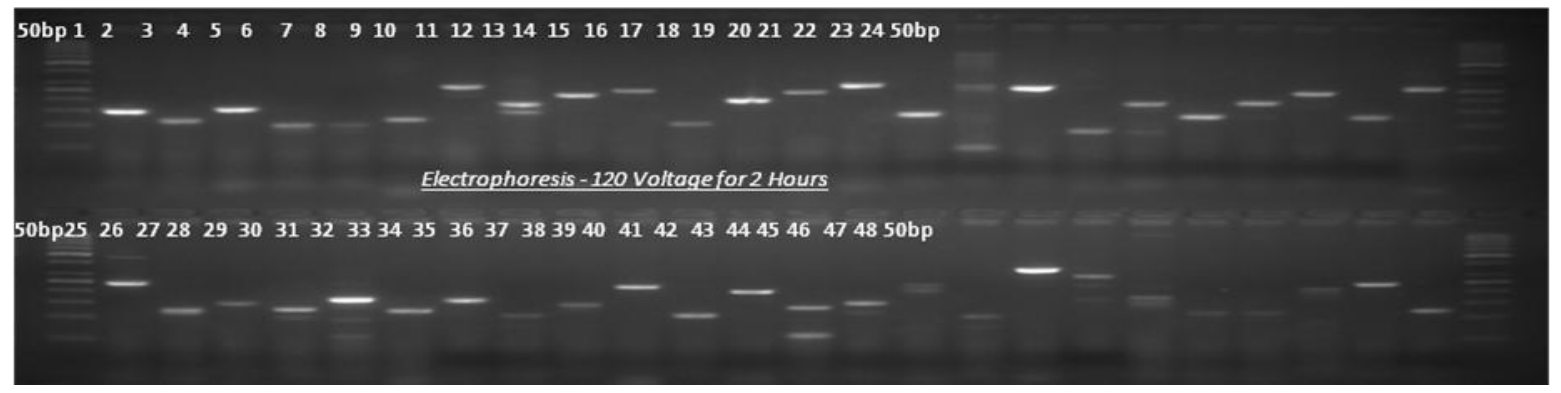

\section{Results and Discussion}

The data depicted in table 1 exhibit that Narendra Lahar posse's distinct, unique and stable morphological trait which differ it from the other high yielding varieties of irrigated ecosystem and land races. DUS test evaluation revealed that Narendra Lahar is medium maturing, maturing, good tillering ability and semi tall rice variety. The basal leaf sheath colour is green in coloration while leaf: anthocynincolration is absent in the variety. Leaf: pubescence of blade surface is very week and colorless auricles were present in the variety. Ligule was present and green in color. The anthocynin coloration of nodes and internodes is absent. It possesses aweless and well exerted panicle. The length of the panicle is medium. The 1000 grain weight of Narendra Lahar is medium and shape of grain is long slender.. It possesses good cooking quality with moderate amylose content $21.56 \%$ having soft gel (Gel consistency - $46 \mathrm{~mm}$ ). Cooked rice of Narendra Lahar is non sticky rice varieties are preferred by the Indian consumers. Gelatinization temperature of the variety was also reported on the basis of alkali spreading value and it was found medium. The band position of prominent SSR markers depicted in table 2 and figure 1 also exhibits distinct and uniqueness of this variety. These SSR markers were used by Shakil et al., (2015) for molecular characterization of modern high yielding rice varieties of Bangladesh. The present study of molecular and morphological characterization of Narendra Lahar will be helpful in the identification of variety and to maintain its genetic purity. This information will be very useful for the persons involved in seed certification and seed production programme.

\section{Acknowledgement}

The authors would like to thanks Dr. T. Ram, Principal Scientist (Plant Breeding), IIRR, Hyderabad for providing DNA fingerprinting facility.

\section{References}

1. Anonymous. 2001. Protection of Plant Varieties and Farmers Right Act (No. 53 of 2001). Dept. of Agriculture and Cooperation Ministry of Agriculture, Govt. of India, Krishi Bhawan, New Delhi.

2. Chakravorty, A., Ghosh, P. D. 2012. Characterization of Landraces of rice following DUS guidelines. Research in Plant Biology 2(6): 30-40.

3. Chakrabarty, S. K., Monika A. Joshi, Yogendra Singh, Aniruddha Maity, Veena Vashisht and Dadlani, M. 2012. Characterization and evaluation of variability in farmers' varieties of rice from West Bengal. Indian journal of Genetics and Plant Breeding 72(2): 136142.

4. Chang TT, Bardenas EA, 1965. The Morphology and Varietal Characteristics 
of the Rice Plant. Tech Bull 4, IRRI, Philippines, pp40.

5. Khush, G. S. 1997. Origin, dispersal and variation of rice. Pl. Mol. Biol. 35: 25-34.

6. Kwon Y.S., Lee J.M., Yi G.-B., (2005) Use of SSR markers to complement tests of distinctiveness, uniformity, and stability (DUS) of pepper (Capsicum annuum L.) varieties, Molecules and Cells, 19 (3), 428-435.

7. Hien NL, Sarhadi WA, Oikawa Y, Hirata Y. 2007. Genetic diversity of morphological responses and the relationships among Asia aromatic rice (Oryza sativa) cultivars. Jpn. Soc.Trop. Ecol. 16: 343-355

8. Hien NL, Yoshihashi T, Sarhadi WA, Hirata Y. 2006. Sensory test for aroma and quantitative analysis of 2-acetyl- 1pyrroline in Asian aromatic rice varieties. Plant Prod. Sci. 9: 294-297

9. INGER - IRRI (1996). Standard Evaluation System for rice. 4thed. IRRI, p. 52.

10. International Rice Research Institute [IRRI]. 1980. Descriptors for Rice Oryza sativa $\mathrm{L}$. The International Rice Research Institute, Manila, Philippines.

11. Patra, B.C. 2000. Collection and characterization of rice genetic resources from Keonjhar district of Orissa. Oryza 34: 324-326.

12. PPV and FRA. 2007. Guidelines for the conduct of test for DUS on rice (Oryza sativa L.). Protection of Plant Varieties and Famrer's Right Authority (PPV\&FRA). Government of India, New Delhi.

13. Raut, V.M. 2003. Qualitative genetics of soyabean review. Soybean Research 1:128.
14. Roy, JK., De, RN., Ghoari, D.P., Panda, A. 1985. Collection and evaluation of genetic resources of rice in India. Phyrtobreedon 1: 1-9.

15. Shobha Rani, N, Shobha Rao, L V, Viraktamath, B.C., Mishra B. 2004. National Guideline for the Conduct of test for Distinctiveness, Uniformity and Stability. Directorate of Rice Research: 613.

16. Singh Y., Singh, U.S. 2008. Genetic diversity analysis rice germplasm using agro-morphological traits. Journal of Plant Genetic Resources 21.(1): 32-37.

17. Song, Q.J., Quigley, C.V., Nelson, R.L., Carter, T.E., Boerma, H.R., Strachan J.L., Crega, P.B. 1999. A selected set of trinucleotide simple sequence repeat markers for soyabean cultivar identification. Plant Variety Seed 12: 207220.

18. Subba Rao, L.V., Shiva Prasad, G., Chiranjivi, M., Chaitanyam U., Surendhar, R. (2013) DUS characterization for farmer's varieties of rice. IOSR Journal of Agriculture and Veterinary Science PP 35-43.

19. Venkatesan K., BHAT K.V. (2015) Microsatellite Marker-Based Molecular Characterization of Small and MediumGrained Aromatic Rice Germplasm of Odisha, India, SABRAO Journal of Breeding and Genetics, 47 (3): 248-259.

20. Shakil S.K., Sultana S., Hassan M.M., Hossain M.M., Ali M.S., Prodhan S.H. (2015) SSR Marker Based Genetic Diversity Analysis of Modern Rice Varieties and Coastal Land Races in Bangladesh, Indian Journal of Biotechnology, 14 (January, 2015): 3341.

\section{How to cite this article:}

Giri, S.P., Alok Pandey, V.N. Singh, D.K. Verma, R.M. Tripathi, Arun Kumar Singh, M.L. Maurya and Singh, R.V. 2017. DUS Characterization of Narendra Lahar: A New High Yielding Rice Variety. Int.J.Curr.Microbiol.App.Sci. 6(11): 5444-5453. doi: https://doi.org/10.20546/ijcmas.2017.611.521 